EPJ Web of Conferences 59, 02018 (2013)

DOI: $10.1051 /$ epjconf/20135902018

(C) Owned by the authors, published by EDP Sciences, 2013

\title{
Burning the DT-plasma with inert impurities and non-cryogenic ICF-target with solid fuel
}

\author{
S.Yu. Gus'kov ${ }^{1, a}$, D.V. Il'in ${ }^{2}$ and V.E. Sherman ${ }^{2}$ \\ 1 P. N. Lebedev Physical Institute of RAS, 119991 Leninskii pr. 53, Moscow, Russia \\ 2 St. Petersburg Institute of Machine Building, 195197 Polyustrovskii pr.14, St. Petersburg, \\ Russia
}

\begin{abstract}
The ignition criterion, ignition energy and gain of DT-plasma of ICF-target in the presence of impurities of light atoms such as beryllium, carbon and lithium at their arbitrary concentration are found. It is shown that the most promising type of non-cryogenic solid thermonuclear fuel is DT-hydride of beryllium (BeDT). It is suggested to apply the targets with such a fuel as: (1) Fast-ignited ICF-target at the ignition energy of 25-50 kJ and compression driver energy of 2-3 MJ; (2) ICF-target spark-ignited by 15-20 MJ heavy ion driver; (3) Spark-ignited target by 5-7 MJ laser as a neutron source for hybrid fusion-fission.
\end{abstract}

\section{INTRODUCTION}

Significant decrease of the neutron yield in modern experiments due to mixing of the fuel and ablator materials can occur at a fraction of impurity of only several percent. The problem of considerable impurity's concentration of about $30 \%$ is important in studying the efficiency of solid non-cryogenic thermonuclear fuel in the form of chemical compounds of hydrogen's isotopes. The inert atoms entering into such compounds should be as light as possible and their concentration should be minimal. The materials satisfying specified above requirements are: DT-hydrides of light metals - BeDT, $\mathrm{Li}_{2} \mathrm{DT}, \mathrm{Li}_{2} \mathrm{BeD}_{2} \mathrm{~T}_{2}, \mathrm{Li}_{2} \mathrm{Be}_{2} \mathrm{D}_{3} \mathrm{~T}_{3}$, and also amine-borane $\mathrm{NT}_{3} \mathrm{BD}_{3}$. Meaning the both problems the ignition and combustion of DT-plasma containing the impurities of carbon $\left.{ }_{6} \mathrm{C}^{12}\right)$, beryllium $\left({ }_{4} \mathrm{Be}^{9}\right)$ and lithium $\left({ }_{3} \mathrm{Li}^{7}\right)$ are investigated. The ignition criterion, ignition energy and gain are defined at arbitrary concentration of impurities for homogeneous plasma, as well as isobaric plasma formed at the conventional spark ignition of ICF-target, including shock ignition [1] and isochoric plasma formed at fast ignition [2]. This paper is the summary of a conference presentation preceding the publication of paper [3], where a number of the results can be found in more detail.

\section{INFLUENCES OF IMPURITY OF LIGHT ATOMS ON ICF-TARGETS IGNITION}

We consider a spherical igniter consisting of a homogeneous, equilibrium (electron an ion temperatures are equal $T_{e}=T_{i}=T$ ) completely ionized plasma containing deuterium and tritium nuclei with equal fractions $x_{F}$ and inert nuclei of different types with fractions $x_{a}$, so that $x_{F}=0.5\left(1-\Sigma x_{a}\right)$. For such an igniter the criterion for ignition which states that the rate of thermonuclear heating of the plasma should exceed the rate of total energy losses due to work of pressure forces, thermal radiation and electron heat conductivity looks like:

$$
\begin{aligned}
8.1 \cdot & 10^{40} \chi_{F} \quad(\rho R)^{2}<\sigma v>\left(\eta_{\alpha}+4 \eta_{n}\right)>6.4 \cdot 10^{22} \chi_{u} \beta T^{3 / 2}(\rho R)+2.8 \cdot 10^{23} \chi_{r} T^{1 / 2}(\rho R)^{2} \\
& +2.6 \cdot 10^{19} \chi_{c} T^{7 / 2}
\end{aligned}
$$

\footnotetext{
ae-mail: guskov@sci.lebedev.ru
}

This is an Open Access article distributed under the terms of the Creative Commons Attribution License 2.0, which permits unrestricted use, distribution, and reproduction in any medium, provided the original work is properly cited. 


\section{EPJ Web of Conferences}

where $\rho$ and $R$ are the density and radius of igniter, $\langle\sigma v\rangle$ is the rate of DT-reaction, $\eta_{\alpha}$ and $\eta_{n}$ are the fractions of $\alpha$-particle and neutron energy deposited in igniter; $\beta$ is the coefficient that links the velocity of piston $u$ with sound velocity in the igniter $V_{s}, u=\beta V_{s}: \beta=0$ for isobaric plasma [1, 4], $\beta=[2 /(\gamma+1)]^{1 / 2} \approx 0.87$ for isochoric plasma (with an adiabatic exponent $\gamma=5 / 3$ ) [4] and $\beta$ is chosen to be 2 for homogeneous plasma [5]. The values of factors of impurity influence on the rates of fusion reaction $\chi_{F}$ and energy losses $\chi_{u}, \chi_{r}$ and $\chi_{c}$ are

$$
\begin{aligned}
\chi_{F} & =\left[\left(\frac{2.5}{\mu_{*}}\right)\left(1-\sum_{a} x_{a}\right)\right]^{2}, \chi_{u}=\left[\left(\frac{2.5}{\mu_{*}}\right)\left(\frac{1+Z_{*}}{2}\right)\right]^{3 / 2}, \chi_{r}=\left[\left(\frac{2.5}{\mu_{*}}\right)^{2}\left(Z^{2}\right)_{*} Z_{*}\right], \\
\chi_{c} & =\left[\frac{5}{Z_{*}+4}\right]
\end{aligned}
$$

where $\mu_{*}, Z_{*},\left(Z^{2}\right)_{*}$ are the average atomic weight, charge and charge square: $\mu_{*}=\left(\mu_{T}+\mu_{D}\right) x_{F}+$ $\Sigma \mu x_{a}, Z_{*}=2 x_{F}+\Sigma Z_{a} x_{a},\left(Z^{2}\right)_{*}=2 x_{F}+\Sigma Z_{a}^{2} x_{a}$. For one-component impurity: $\mu_{*}=2.5+\left(\mu_{a}-\right.$ $2.5) x, Z_{*}=1+\left(Z_{a}-1\right) x,\left(Z^{2}\right)_{*}=1+\left(Z_{a}^{2}-1\right) x$. If impurities are absent $\left(x_{a}=0\right) \chi_{F}=\chi_{u}=\chi_{r}=$ $\chi_{c}=1$.

Presence of impurity weakly changes an $\alpha$-particle energy fraction deposited in plasma. Besides, ignition occurs at $\rho R \ll 4-6 \mathrm{~g} / \mathrm{cm}^{2}$ when neutron energy deposition is small. Negative influence of impurities on ignition is connected with decreasing the reaction rate and increasing the rate of radiation energy losses. At 30\% impurity of carbon, $\chi_{F}$ decreases 9 times, and $\chi_{r}$ increases more than 6 times in comparison with pure DT-plasma. At the same time $\chi_{u}$ and $\chi_{c}$ are in the range 1.2-1.4. Estimation of minimal ignition temperature comes from equality of rates of heating and radiation energy losses. For one-component impurity $T_{i g(\min )}=3.7\left[\left(Z^{2}\right)_{*} Z_{*} /(1-x)^{2}\right]^{2 / 5} \mathrm{keV}$. For pure DT-plasma $T_{i g(\min )} \approx$ $4 \mathrm{keV}$. For example, 30\% impurity of carbon and beryllium increases $T_{i g(\min )}$, respectively, to $19.4 \mathrm{keV}$ and $12.1 \mathrm{keV}$. From an equality of the greatest possible heating rate (at $T=60 \mathrm{keV}, \eta_{\alpha}=\eta_{n}=1$ ) and rate of radiation energy losses $\left(150 \chi_{F}=\chi_{r}\right)$, the estimation of limiting impurity's concentration at which ignition isn't possible at any values of $\rho R$ and $T$ follows: $42 \%$ for carbon, $59 \%$ for a beryllium and $69 \%$ for lithium. The approximate solution of ignition-criterion (1) gives the values of minimal ignition-parameter $\rho R$ and corresponding plasma energy

1. $\beta=0\left(T_{i g}=10 \mathrm{keV},(\rho R)_{\text {min }} \leq 1 \mathrm{~g} / \mathrm{cm}^{2}: x_{B e} \leq 0.3, x_{C} \leq 0.18\right)$

$$
\begin{aligned}
(\rho R)_{\min } & \simeq 0.18\left[\frac{\left(\frac{\mu_{*}}{2.5}\right)^{2}\left(\frac{5}{4+Z_{*}}\right)}{(1-x)^{2}-0.09\left(Z^{2}\right)_{*} Z_{*}}\right]^{1 / 3}, \frac{g}{c m^{2}} ; \\
E_{i g} & \simeq 7.1 \cdot 10^{3}\left[\frac{\left(\frac{\mu_{*}}{2.5}\right)\left(\frac{Z_{*}+1}{Z_{*}+4}\right)}{(1-x)^{2}-0.09\left(Z^{2}\right)_{*} Z_{*}}\right] \rho_{100}^{-2}, J
\end{aligned}
$$

2. $\beta=0,87$ and $\beta=2\left(T_{i g}=15 \mathrm{keV},(\rho R)_{\min } \leq 1 \mathrm{~g} / \mathrm{cm}^{2}: x_{B e} \leq 0.2, x_{C} \leq 0.12\right)$

$$
\begin{aligned}
(\rho R)_{\min } & \simeq 0.35\left[\frac{\beta\left(\frac{\mu_{*}}{2.5}\right)^{1 / 2}\left(\frac{1+Z_{*}}{2}\right)^{3 / 2}}{(1-x)^{2}-0.06\left(Z^{2}\right)_{*} Z_{*}}\right]^{1 / 2}, \frac{g}{c m^{2}} ; \\
E_{i g} & \simeq 3.1 \cdot 10^{4}\left[\frac{\beta\left(\frac{2.5}{\mu_{*}}\right)^{1 / 6}\left(\frac{Z_{*}+1}{2}\right)^{13 / 6}}{(1-x)^{2}-0.06\left(Z^{2}\right)_{*} Z_{*}}\right]^{3 / 2} \rho_{100}^{-2}, J .
\end{aligned}
$$

Here $\rho_{100}$ is measured in units of $100 \mathrm{~g} / \mathrm{cm}^{3}$ Presence of impurity leads to growth the both $(\rho R)_{\text {min }}$ and corresponding ignition temperature $T_{i g}$ in comparison with pure DT-plasma. For pure DT-plasma: 
$(\rho R)_{\min } \approx 0.18 \mathrm{~g} / \mathrm{cm}^{2}$ at $T_{i g} \approx 7 \mathrm{keV}$ in the case of an isobaric plasma $(\beta=0) ;(\rho R)_{\min } \approx 0.32 \mathrm{~g} / \mathrm{cm}^{2}$ at $T_{i g} \approx 10 \mathrm{keV}$ in the case of isochoric plasma $(\beta=0.87)$ and $(\rho R)_{\min } \approx 0.48 \mathrm{~g} / \mathrm{cm}^{2}$ at $T_{i g} \approx 12 \mathrm{keV}$ in the case of a homogeneous plasma $(\beta=2)$. At $10 \%$ impurity, $(\rho R)_{\min }$ increases by about 1.5 times in the case of a beryllium impurity and by about 2 times in the case of carbon impurity for all plasma configurations. The ignition temperature increases essentially less, namely, in 1.1-1.3 times. At 10\% impurity, the ignition energy growth makes about 3.5 times in the case of beryllium impurity and about 8 times in the case of carbon impurity.

\section{INFLUENCES OF IMPURITY OF LIGHT ATOMS ON ICF-TARGETS BURNING}

Thermonuclear gain of inhomogeneous plasma with igniter's mass $M_{i g}$ much less than the total fuel mass $M$ is approximately expressed through the gain $G_{0}$ of homogeneous plasma with the mass equal to the total fuel mass $M$, temperature equal to the igniter temperature $T_{i g}$, and density equal to the density of cold fuel $\rho_{c}$

$$
G \approx \frac{G_{0} M T_{i g}}{M_{i g} T_{i g}+\left(M-M_{i g}\right) T_{c}}
$$

where $T_{c}$ is the temperature of cold fuel. Gain of homogeneous plasma containing the impurities is

$$
G_{0}=\frac{\varepsilon_{r} g}{3 T_{i g}}\left(\frac{1-\sum_{a} x_{a}}{1+Z_{*}}\right)
$$

where $\varepsilon_{r}=17.6 \mathrm{MeV}$ is energy realized in DT-reaction, $g=n_{F 0}-n_{F} / n_{F}$ is burning out of hydrogen isotopes. Solution of the equation for burning out of DT-plasma with one-component impurity gives

$$
\begin{aligned}
& g \simeq \frac{\rho R}{\rho R+2.9\left\{\left[2+\left(Z_{a}-1\right) x\right]\left[2.5+\left(\mu_{a}-2.5\right) x\right]\right\}^{1 / 2}}, x \leq x_{c} ; \\
& g \simeq \frac{\rho R}{\rho R+2.9 \frac{\left[2+\left(Z_{a}-1\right) x\right]^{3}\left[2.5+\left(\mu_{a}-2.5\right) x\right]^{1 / 2}}{[5(1-x)]^{5 / 2}}}, x_{c} \leq x \leq 1 .
\end{aligned}
$$

For all types of impurities at their concentration smaller than the critical values $x_{c}=3 /\left(Z_{a}+4\right)$ burning out practically doesn't change with increasing the impurities concentration, remaining equal, approximately, to $g=\rho R /(\rho R+6.5)$ for pure DT-plasma. Weak growth of burning out with impurities concentration growth in the region $x<x_{c}$ is connected with increasing the confinement time. At $x>x_{c}$ the sharp decreasing the burning out with growth of impurities concentration begins. It is connected with decreasing the temperature of combustion less than $20-30 \mathrm{keV}$. Critical concentrations are: 0.43 for lithium, 0.37 for beryllium and 0.3 for carbon. In fig. 1 the dependencies are shown of burning out of homogeneous plasma with $\rho_{D T} R=3 \mathrm{~g} / \mathrm{cm}^{2}$ on light impurities concentration calculated on the base of analytical model and numerically by using the code TERA [6] at $T_{i g}=15 \mathrm{keV}$. At $x=x_{c}$ thermonuclear gain decreases in 2-2.5 times in comparison with pure DT: from 62 down to 30-25.

\section{IGNITION AND COMBUSTION OF SOLID NON-CRYOGENIC FUEL}

DT-hydride of beryllium (BeDT) is the most suitable material as a solid non-cryogenic fuel. BeDT has the highest relation $\chi_{F} / \chi_{r}=0.038\left(\chi_{F}=0.13, \chi_{r}=3.44\right)$ among all materials listed in the introduction. In fig. 1 ignition $\rho R-T$ areas for different configurations of BeDT and DT-plasmas are shown. For the spark ignited BeDT-plasma, according to the data of fig. $1, T_{i g}=15 \mathrm{keV}$ and $(\rho R)_{\min }=0.9 \mathrm{~g} / \mathrm{cm}^{2}$, hence formula (3) gives for igniter's nergy $E_{i g} \approx 4.2 \cdot 10^{9} / \rho_{i g}^{2} \mathrm{~J}$, and for isobaric plasma at $\rho R>(\rho R)_{i g}$ 

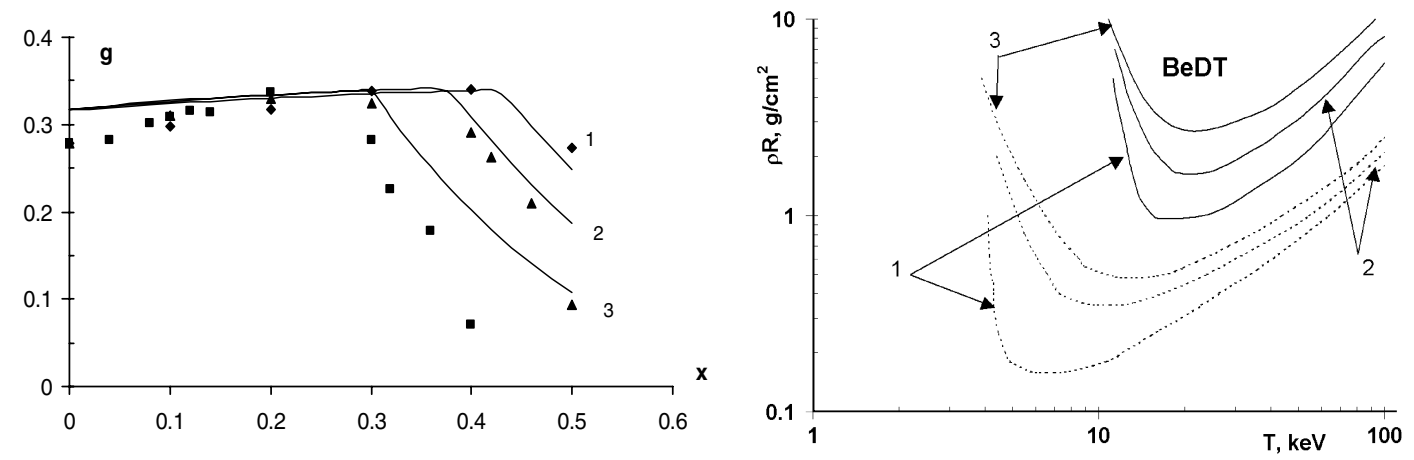

Figure 1. (left). Burning out of DT-plasma with $\rho_{D T} R=3 \mathrm{~g} / \mathrm{cm}^{2}$ vs concentration of lithium (1), beryllium (2) and carbon (3). The numerical data are shown by rhombuses (lithium), triangles (beryllium), and squares (carbon). (right). Ignition curves for different configurations of BeDT-plasma (solid lines) and pure DT-plasma (dashed lines). Curves 1,2,3 corresponds to isobaric, isochoric and homogeneous plasmas.

and $\rho_{c} \gg \rho_{i g}$ total initial fuel's energy and gain, according to (5), are

$$
E \approx \frac{4.2 \cdot 10^{9}}{\rho_{i g}^{2}}\left[1+3.3(\rho R-0.9) \rho_{i g} / \rho_{c}\right], \mathrm{J} ; G \approx 86\left(\frac{\rho R}{\rho R+10.8}\right)\left[\frac{1+3.3(\rho R-0.9)}{1+3.3(\rho R-0.9) \rho_{i g} / \rho_{c}}\right] .
$$

The gain grows with growth of the relation of densities $\rho_{c} / \rho_{i g}$. For $\rho R=6 \mathrm{~g} / \mathrm{cm}^{2}$ and $\rho_{c} / \rho_{i g}=10$ $\left(\rho_{c}=1000 \mathrm{~g} / \mathrm{cm}^{3}, \rho_{i g}=100 \mathrm{~g} / \mathrm{cm}^{3}\right)$ gain makes $G \approx 200$ at the energy $E \approx 1 \mathrm{MJ}$. These gains could be enough for heavy ion fusion at the driver energy of 15-20 MJ. For laser fusion such gains are insufficient. However, the BeDT-target with gain 20-30 can be used as a laser-produced source of neutrons in the hybrid fusion-fission reactor at the laser energy 5-7 MJ .

For the fast ignited BeDT-plasma, according to the data of fig. $1, T_{i g}=20 \mathrm{keV}$ and $(\rho R)_{\min }=$ $1.5 \mathrm{~g} / \mathrm{cm}^{2}$, hence formula (4) gives for igniter's energy $E_{i g} \approx 2.6 \cdot 10^{10} / \rho_{i g}^{2} \mathrm{~J}$, and for isochoric plasma at $\rho R \gg(\rho R)_{i g}$ and $\rho_{c}=\rho_{i g}$ total initial fuel's energy and gain are

$$
E \approx \frac{2.6 \cdot 10^{10}}{\rho^{2}}\left[1+5.8 \cdot 10^{-5}(\rho R)^{3} \rho^{2 / 3}\right], \mathrm{J} ; G \approx 64\left(\frac{\rho R}{\rho R+10.8}\right)\left[\frac{1+0.3(\rho R)^{3}}{1+5.8 \cdot 10^{-5}(\rho R)^{3} \rho^{2 / 3}}\right]
$$

For $\rho R=6 \mathrm{~g} / \mathrm{cm}^{2}$ and the density in the range $\rho=300-1000 \mathrm{~g} / \mathrm{cm}^{3}$ gain makes $G \approx 1000-600$ at the initial plasma energy $E \approx 450-60 \mathrm{~kJ}$ and igniter's energy $E_{i g} \approx 280-25 \mathrm{~kJ}$, respectively. Thus, the target with BeDT-fuel can be used as an ICF-target fast ignited by laser-produced beams of electrons or ions with energy $25-50 \mathrm{~kJ}$ at the fuel compressed to the density of $1000-700 \mathrm{~g} / \mathrm{cm}^{3}$ or fast ignited by heavy ion beam with energy of $100-280 \mathrm{~kJ}$ at the fuel compressed to the density of $500-300 \mathrm{~g} / \mathrm{cm}^{3}$.

\section{CONCLUSION}

The ignition criterion, ignition energy and gain are found for DT-plasmas containing light impurities at arbitrary concentration. It is suggested to apply non-cryogenic targets with solid BeDT-fuel as ICFtarget for fast ignition, heavy ion fusion target for spark ignition, and spark-ignited target as a laserproduced neutron source for hybrid fusion-fission approach. 
The work was supported by the Ministry of Education and Science of the Russian Federation under the program "Development of the Scientific Potential of Higher Education for 2011" (Project no. 2.1.1/12138) and by the Russian Foundation for Basic Research (Project no. 10-02-92104-à-JR).

\section{References}

[1] V.A. Scherbakov, Sov. J. Plasma Phys. 9, 240 (1983)

[2] N.G. Basov, S.Yu.Gus'kov, L.P. Feoktistov, J. Soviet Laser Research 13, 396 (1992)

[3] S.Yu. Gus'kov, D.V. Il'in, V.E.Sherman, Plasma Physics Reports 37, 1020 (2011)

[4] S. Atzeni and J. Meyer-ter-Vehn, The physics of inertial fusion (Oxford University Press, 2004)

[5] V.B. Rozanov, C. P. Verdon., M. Decroisette et al, Inertial confinement target physics, In the Book: Energy from inertial fusion (IAEA, Vienna, 1995)

[6] S.Yu. Gus'kov, D.V. Il'in, A.A. Levkovsky et al, Laser and Particle Beams 16, 129 (1998) 